\title{
Growth Analysis of Pigeon Pea and Chickpea in Marathwada Region of Maharashtra State, India
}

\author{
N.R. Dhokar*, S.S. More and R.D. Shelke \\ Department of Agricultural Economics, College of Agriculture, Latur, \\ Vasantrao Naik Marathwada Krishi Vidyapeeth, Parbhani 431402 (M.S.), India \\ *Corresponding author
}

Keywords

Marquardt algorithm, Pigeon pea, Chickpea, Marathwada, Maharashtra

Article Info

Accepted: 12 July 2018 Available Online: 10 August 2018

\section{A B S T R A C T}

The paper examines the growth analysis of area, production and productivity of pigeon pea and chickpea in Marathwada region of Maharashtra State. Compound growth rate was estimated by fitting non-linear model to the area, production and productivity data for the period from 1986-87 to 2015-16. The fitted model was analyzed using Marquardt algorithm. The compound growth rates were tested for their significance. The results shows that, the compound growth rate in area, production and productivity of pigeon pea was positive and significant at 1 per cent level of significance during overall study period except in Aurangabad and Parbhani district in these districts growth in pigeon pea area was decreased. During the overall period, the growth performance of pigeon pea in Marathwada region was positive and significance in area, production and productivity. The highest compound growth rate in area of pigeon pea was observed in Hingoli district. Highest production and productivity growth rate was seen in Latur district. The area, production and yield of chickpea were consistently increased during the study period. Improvement in the productivity of pulses was notable feature in all the districts of Marathwada region. Expansion of area enhancement in productivity and improvement in productivity was observed in major pulses in the region. This was because of implementation of pulse development programme by government of India in the state.

\section{Introduction}

Pulses have a unique role to play in the global nitrogen cycle, pulse fix atmospheric nitrogen in soils. The introduction of pulses into crop rotations actively helps to fix nitrogen in the soil and also reduce the greenhouse gas emission to the environment from subsequent crops therefore it maximizes the environmental benefits.
Pulses are grown in more than 171 countries. The world's major producers of pulses are India (23.1 per cent), China (12.08 per cent), Myanmar (7.57 per cent), Canada (6.7 per cent) and Brazil (4.03 per cent) which together account for half of the global output (Shingne et al., 2017). India is largest producer (25 per cent of global production), consumer (27 per cent of world consumption) and importer (14 per cent) of pulses in the world (Chavan et al., 2017). Pulses account 
for around 20 per cent of the area under foodgrains and contribute around 7-10 per cent of the total food grains production in the country. Though pulses are grown in both Kharif and Rabi seasons, Rabi pulses contribute more than 60 per cent of the total pulse production. Gram is the most dominant pulses having a share of around 40 per cent in the total production followed by Arhar at 1520 per cent and Black gram and Green gram at around 8-10 per cent each. The major pulses producing states are Madhya Pradesh, Maharashtra, Rajasthan, Uttar Pradesh, Karnataka and Andhra Pradesh which together accounts for about 80 per cent of the total production.

The pulses in India are grown in semi-arid areas which face high rainfall variability adding to high instability and low productivity. Best parcel of lands with irrigation facility is usually reserved for other crops by farmers. The progress of pulses has always been lukewarm in spite of the overall impressive growth of Indian agriculture. The government has focused on improving pulse production through various programmes like in 1967 it established the All India Coordinated Pulses Improvement Project (AICRIP), which was later elevated to the Indian Institute of Pulses Research (IIPR). Pulses also have received significant attention in different five-year plans, including the Intensive Pulses District Program launched during the Fourth Five-Year Plan (19691974), National Pulses Development Program launched during the Seventh Five-Year Plan (1985-89), Technology Mission on pulses 1986 and a special food grain production program launched in 1988-1989, National Pulses Development Project in 1990-91, Integrated Scheme of Oilseeds, Pulses, Oil palm and Maize in 2004 (ISOPOM). National Food Security Mission in 2007-08 being implemented in 171 identified districts of 14 major pulses growing states and A3P i.e. Accelerated Pulses Production Programme
(2010) but supply remain down the line compared to the demand and country has to heavily relies on imports to bridge the supplydemand gap. The growth in production and productivity of pulses has lagged behind the population growth rate which resulted into a decline in per capita availability of pulses from $66 \mathrm{~g}$ /day during triennium ending (TE) 1965 to 47.20 g/day during 2016 against Indian Council of Medical Research (ICMR) norms of $40 \mathrm{~g} /$ day (Chavan et al., 2017).

Maharashra is one of the major pulse productivity states. Government of India has all the pulse development programme viz; Intensive pulses district programme, National pulse development programme, technology mission of pulses, ISOPOM, NFSM and A3P which aims to improve the pulse production.

Inspite these programme, there is gap between demand and supply of pulses of national level. So, this particular study was under taken to know the effect of there pulses development programme (which were implemented in Maharashtra) on performance of the pulses in the state. The specific objective of the study was to estimate growth rates of area, production and yield of major pulse crops in Marathwada region.

\section{Materials and Methods}

\section{Collection of data}

Marathwada region was selected purposively for the study. The entire eight district of Marathwada region was selected. The analysis is primarily based on secondary data related to area, production and productivity of pulses. The growth in area, production and productivity of two major pulses, viz; Pigeon pea and Chickpea were studied. The time series secondary data were collected from various sources viz; Epitome of Agriculture Part II published by Government of Maharashtra. The official website of 
Department of Agriculture, Government. of Maharashtra. The data were collected for the period of 30 years i.e. from 1986-87 to 201516. The entire study was split into three sub periods. The sub period was framed as Period-I: $1986-87$ to 1995-96, Period-II: 1996-97 to 2005-06, Period-III: 2006-07 to 2015-16 and Overall Period: 1986-87 to 2015-16. Specifically data were collected as Government of India has launched from 1986-87, Technology Mission on Pulses during 1986 to increase the production of pulses, to reduce import and to achieve selfsufficiency in pulses.

\section{Growth rate}

The compound growth rate was computed based on its fit using non -linear models, especially, the exponential model. Conventionally, the compound growth rates were estimated after converting the growth model to semi-log form and estimated through Ordinary Least Square (OLS) technique assuming multiplicative error term. However, there are several problems associated with this technique including the difficulty in estimating standard error of estimates of original parameters. Hence, a non-linear estimation technique for solving exponential model assuming additive error terms was used to estimate the compound growth rates.

$\mathrm{y}_{\mathrm{t}}=$ constant $*(1+\mathrm{CGR})^{\mathrm{t}}+\epsilon_{\mathrm{t}} \ldots$

Where,

$\mathrm{y}_{\mathrm{t}}$ is the time series data for area/production/yield for year $\mathrm{t}$, $t$ is the time trends for years of interest, $\epsilon_{t}$ is the error term and CGR is compound growth rate for the period under consideration.

The Marquardt algorithm was used to estimate the parameters of equation. The data were smoothened by taking three year moving average to remove bias if any, induced by the outliers. The significance of regression coefficients were tested by applying standard ' $t$ ' test procedure. The data were analyzed with the help of Statistical Analysis System (SAS) software package, Version 9.3.

\section{Results and Discussion}

\section{Pigeon pea}

The growth in area, production and yield of two major crops viz; pigeon pea and chickpea was assessed and presented in table 1 and 2 . The annual compound growth rate (CGR) in area of pigeon pea crop was 2.95, 0.31, 2.23 and 1.39 per cent per annum during three sub periods and overall period at Marathwada region of Maharashtra State (Table 1). It was statistically significant at 1 per cent level of significance. This shows that, maximum growth in area of pigeon pea crop has been occurred during first period (1986-87 to 199596). Yield of pigeon crop has increased during overall period significantly mixed trend was seen during three sub period. Similar results were reported by Brahmaprakash and Srivastava (1998). During overall period productivity under pigeon crop was 2.62 per cent per annum. It was statistically significant at 1 per cent level of significance. Positive and significant trend in production of pigeon pea crop was also seen in overall period. During this period CGR of production was highest 3.97 per cent per annum. Similar results were obtained by Asthurkar and Kolhal (1993).

The CGR of production during three sub periods were $1.01,2.73$ and -0.78 per cent, respectively. The district level results shows that, in first sub period of study (1986-87 to 1995-96), all the districts has shown positive trend in area of pigeon pea but high CGR i.e. more than 3 per cent was recorded in Aurangabad, Osmanabad and Parbhani districts. 
Table.1 Compound growth rates of area, production and productivity of pigeon pea in Marathwada region of Maharashtra State

\begin{tabular}{|c|c|c|c|c|c|c|c|c|c|c|c|c|}
\hline \multirow{3}{*}{$\begin{array}{l}\text { District } \\
\text { Period } \\
\end{array}$} & \multicolumn{3}{|c|}{ Period-I } & \multicolumn{3}{|c|}{ Period-II } & \multicolumn{3}{|c|}{ Period-III } & \multicolumn{3}{|c|}{ Overall } \\
\hline & \multicolumn{3}{|c|}{$1986-87$ to $1995-96$} & \multicolumn{3}{|c|}{$1996-97$ to $2005-06$} & \multicolumn{3}{|c|}{ 2006- 07 to $2015-16$} & \multicolumn{3}{|c|}{$1986-87$ to $2015-16$} \\
\hline & $\mathbf{A}$ & $\mathbf{P}$ & $\mathbf{Y}$ & $\mathbf{A}$ & $\mathbf{P}$ & $\mathbf{Y}$ & $\mathbf{A}$ & $\mathbf{P}$ & $\mathbf{Y}$ & $\mathbf{A}$ & $\mathbf{P}$ & $\bar{Y}$ \\
\hline Aurangabad & $\begin{array}{c}6.18 * * \\
(1.42)\end{array}$ & $\begin{array}{l}4.46^{N S} \\
(2.84)\end{array}$ & $\begin{array}{c}-0.68^{\mathrm{NS}} \\
(2.86)\end{array}$ & $\begin{array}{c}-2.37 * * \\
(0.66)\end{array}$ & $\begin{array}{c}-1.27^{\mathrm{NS}} \\
(2.45)\end{array}$ & $\begin{array}{l}1.80^{\mathrm{NS}} \\
(2.31)\end{array}$ & $\begin{array}{c}-1.37^{\mathrm{NS}} \\
(1.46)\end{array}$ & $\begin{array}{c}-5.45^{\mathrm{NS}} \\
(2.51)\end{array}$ & $\begin{array}{c}-4.51 * * \\
(1.08)\end{array}$ & $\begin{array}{c}-0.17^{\mathrm{NS}} \\
(0.33)\end{array}$ & $\begin{array}{c}3.67 * * \\
(0.81)\end{array}$ & $\begin{array}{c}4.08 * * \\
(0.66)\end{array}$ \\
\hline Jalna & $\begin{array}{l}1.65 * \\
(0.61)\end{array}$ & $\begin{array}{c}-1.75^{\mathrm{NS}} \\
(1.86)\end{array}$ & $\begin{array}{c}-3.45^{\mathrm{NS}} \\
(2.30)\end{array}$ & $\begin{array}{c}2.16 * * \\
(0.34)\end{array}$ & $\begin{array}{c}4.76 * * \\
(1.35)\end{array}$ & $\begin{array}{l}2.87^{\mathrm{NS}} \\
(1.42)\end{array}$ & $\begin{array}{c}-1.23^{\mathrm{NS}} \\
(0.78)\end{array}$ & $\begin{array}{c}-7.36 * * \\
(1.02)\end{array}$ & $\begin{array}{c}-5.91 * * \\
(0.97)\end{array}$ & $\begin{array}{c}1.05 * * \\
(0.15)\end{array}$ & $\begin{array}{c}3.33 * * \\
(0.63)\end{array}$ & $\begin{array}{c}2.37 * * \\
(0.54)\end{array}$ \\
\hline Beed & $\begin{array}{l}1.85^{*} \\
(0.77)\end{array}$ & $\begin{array}{l}1.50^{\mathrm{NS}} \\
(2.37)\end{array}$ & $\begin{array}{c}0.12^{\mathrm{NS}} \\
(2.15)\end{array}$ & $\begin{array}{c}-0.97^{*} \\
(0.40)\end{array}$ & $\begin{array}{l}-5.42^{*} \\
(1.69)\end{array}$ & $\begin{array}{l}0.31^{\mathrm{NS}} \\
(2.75)\end{array}$ & $\begin{array}{l}1.49 * \\
(0.57)\end{array}$ & $\begin{array}{c}-7.14 * * \\
(1.20)\end{array}$ & $\begin{array}{c}-7.96 * * \\
(1.20)\end{array}$ & $\begin{array}{c}0.62 * * \\
(0.15)\end{array}$ & $\begin{array}{c}1.77 * * \\
(0.48)\end{array}$ & $\begin{array}{l}1.51^{*} \\
(0.61)\end{array}$ \\
\hline Latur & $\begin{array}{l}1.71 * \\
(0.65)\end{array}$ & $\begin{array}{l}3.63^{\mathrm{NS}} \\
(3.24)\end{array}$ & $\begin{array}{c}2.27^{\mathrm{NS}} \\
(3.20)\end{array}$ & $\begin{array}{c}-0.47^{\mathrm{NS}} \\
(0.49)\end{array}$ & $\begin{array}{l}3.08^{\mathrm{NS}} \\
(2.25)\end{array}$ & $\begin{array}{l}3.71^{\mathrm{NS}} \\
(2.41)\end{array}$ & $\begin{array}{c}6.20 * * \\
(0.33)\end{array}$ & $\begin{array}{l}6.71^{\mathrm{NS}} \\
(3.37)\end{array}$ & $\begin{array}{l}2.50^{\mathrm{NS}} \\
(2.93)\end{array}$ & $\begin{array}{c}2.26 * * \\
(0.21)\end{array}$ & $\begin{array}{c}6.62 * * \\
(0.64)\end{array}$ & $\begin{array}{c}4.30 * * \\
(0.55)\end{array}$ \\
\hline Osmanabad & $\begin{array}{l}4.02 * \\
(1.44)\end{array}$ & $\begin{array}{l}1.16^{\mathrm{NS}} \\
(4.70)\end{array}$ & $\begin{array}{c}-1.55^{\mathrm{NS}} \\
(4.23)\end{array}$ & $\begin{array}{c}0.70^{\mathrm{NS}} \\
(0.33)\end{array}$ & $\begin{array}{c}-0.19^{\mathrm{NS}} \\
(2.95)\end{array}$ & $\begin{array}{c}-0.63^{N S} \\
(2.87)\end{array}$ & $\begin{array}{c}0.39^{\mathrm{NS}} \\
(1.11)\end{array}$ & $\begin{array}{c}-1.30^{\mathrm{NS}} \\
(1.86)\end{array}$ & $\begin{array}{c}-1.88^{\mathrm{NS}} \\
(1.87)\end{array}$ & $\begin{array}{l}1.30 * * \\
(0.20)\end{array}$ & $\begin{array}{c}4.65 * * \\
(0.66)\end{array}$ & $\begin{array}{c}3.46^{* * *} \\
(0.63)\end{array}$ \\
\hline Nanded & $\begin{array}{l}1.81 * * \\
(0.21)\end{array}$ & $\begin{array}{l}1.46^{\mathrm{NS}} \\
(2.70)\end{array}$ & $\begin{array}{c}-0.15^{\mathrm{NS}} \\
(2.74)\end{array}$ & $\begin{array}{l}2.09 * * \\
(0.24)\end{array}$ & $\begin{array}{l}5.48 * \\
(1.91)\end{array}$ & $\begin{array}{l}3.59^{\mathrm{NS}} \\
(1.87)\end{array}$ & $\begin{array}{c}3.06 * * \\
(0.14)\end{array}$ & $\begin{array}{c}-2.51^{\mathrm{NS}} \\
(1.35)\end{array}$ & $\begin{array}{c}-4.97 * * \\
(1.20)\end{array}$ & $\begin{array}{l}1.96 * * \\
(0.08)\end{array}$ & $\begin{array}{c}1.85 * * \\
(0.50)\end{array}$ & $\begin{array}{c}0.37^{\mathrm{NS}} \\
(0.53)\end{array}$ \\
\hline Parbhani & $\begin{array}{l}3.14 * * \\
(0.63)\end{array}$ & $\begin{array}{c}-1.26^{\mathrm{NS}} \\
(2.01)\end{array}$ & $\begin{array}{c}-4.42 * \\
(1.80)\end{array}$ & $\begin{array}{c}-4.88 * * \\
(1.10)\end{array}$ & $\begin{array}{c}-3.16^{\mathrm{NS}} \\
(1.84)\end{array}$ & $\begin{array}{l}1.17^{\mathrm{NS}} \\
(1.99)\end{array}$ & $\begin{array}{c}2.11 * * \\
(0.22)\end{array}$ & $\begin{array}{c}-0.32^{\mathrm{NS}} \\
(1.74)\end{array}$ & $\begin{array}{c}-2.15^{\mathrm{NS}} \\
(1.59)\end{array}$ & $\begin{array}{c}-0.24^{\mathrm{NS}} \\
(0.25)\end{array}$ & $\begin{array}{c}0.21^{\mathrm{NS}} \\
(0.39)\end{array}$ & $\begin{array}{c}0.38^{\mathrm{NS}} \\
(0.40)\end{array}$ \\
\hline Hingoli & NA & NA & NA & $\begin{array}{c}-1.01^{\mathrm{NS}} \\
(0.42)\end{array}$ & $\begin{array}{l}8.23 * * \\
(1.55)\end{array}$ & $\begin{array}{l}9.44 * * \\
(1.39)\end{array}$ & $\begin{array}{c}6.02 * * \\
(0.63)\end{array}$ & $\begin{array}{l}0.53^{\mathrm{NS}} \\
(2.10)\end{array}$ & $\begin{array}{c}-5.53 * * \\
(1.53)\end{array}$ & $\begin{array}{c}3.43 * * \\
(0.54)\end{array}$ & $\begin{array}{c}4.78 * * \\
(1.11)\end{array}$ & $\begin{array}{c}2.00^{\mathrm{NS}} \\
(1.18)\end{array}$ \\
\hline Marathwada & $\begin{array}{c}2.95 * * \\
(0.73)\end{array}$ & $\begin{array}{l}1.01^{\mathrm{NS}} \\
(2.53)\end{array}$ & $\begin{array}{c}-1.57^{\mathrm{NS}} \\
(2.50)\end{array}$ & $\begin{array}{c}0.31^{\mathrm{NS}} \\
(0.29)\end{array}$ & $\begin{array}{l}2.73^{N S} \\
(1.73)\end{array}$ & $\begin{array}{l}2.59^{\mathrm{NS}} \\
(1.72)\end{array}$ & $\begin{array}{c}2.23 * * \\
(0.32)\end{array}$ & $\begin{array}{c}-0.78^{\mathrm{NS}} \\
(1.40)\end{array}$ & $\begin{array}{c}-2.55^{\mathrm{NS}} \\
(1.29)\end{array}$ & $\begin{array}{l}1.39 * * \\
(0.11)\end{array}$ & $\begin{array}{c}3.97 * * \\
(0.44)\end{array}$ & $\begin{array}{l}2.62 * * \\
(0.43)\end{array}$ \\
\hline Maharashtra & $\begin{array}{l}3.79 * * \\
(0.45)\end{array}$ & $\begin{array}{l}3.82 * \\
(1.48)\end{array}$ & $\begin{array}{c}0.12^{\mathrm{NS}} \\
(1.53)\end{array}$ & $\begin{array}{c}0.34^{\mathrm{NS}} \\
(0.16)\end{array}$ & $\begin{array}{l}1.71^{\mathrm{NS}} \\
(1.08)\end{array}$ & $\begin{array}{l}1.43^{\mathrm{NS}} \\
(1.07)\end{array}$ & $\begin{array}{c}1.07 * \\
(0.38)\end{array}$ & $\begin{array}{c}-1.22^{\mathrm{NS}} \\
(1.36)\end{array}$ & $\begin{array}{c}-2.05^{\mathrm{NS}} \\
(1.15)\end{array}$ & $\begin{array}{l}1.17 * * \\
(0.11)\end{array}$ & $\begin{array}{c}2.14 * * \\
(0.28)\end{array}$ & $\begin{array}{l}1.06^{* * *} \\
(0.26)\end{array}$ \\
\hline
\end{tabular}

Note: Figures in brackets indicate standard error, ${ }^{*}$ Significant at 5 per cent level, ${ }^{* *}$ Significant at 1 per cent level, NS- Non significant 
Table.2 Compound growth rates of area, production and productivity of chickpea in Marathwada region of Maharashtra State

\begin{tabular}{|c|c|c|c|c|}
\hline $\begin{array}{l}\text { District } \\
\text { Period }\end{array}$ & Period-I & Period-II & Period-III & Overall \\
\hline 2015-16 & 1986-87 to $1995-96$ & $1996-97$ to $2005-06$ & 2006- 07 to 2015-16 & 1986-87 to \\
\hline
\end{tabular}

\begin{tabular}{|c|c|c|c|c|c|c|c|c|c|c|c|c|}
\hline Aurangabad & $\begin{array}{l}4.34^{*} \\
(1.64)\end{array}$ & $\begin{array}{l}\text { 8.50* } \\
(3.25)\end{array}$ & $\begin{array}{l}\text { 6.52* } \\
(2.15)\end{array}$ & $\begin{array}{c}-\mathbf{- 0 . 8 8} 8^{\mathrm{NS}} \\
(0.80)\end{array}$ & $\begin{array}{c}-0.20^{\mathrm{NS}} \\
(0.95)\end{array}$ & $\begin{array}{c}\mathbf{0 . 6 3} 3^{\mathrm{NS}} \\
(\mathbf{0 . 8 1})\end{array}$ & $\begin{array}{c}-3.11^{*} \\
(1.06)\end{array}$ & $\begin{array}{c}-7.66 * * \\
(2.04)\end{array}$ & $\begin{array}{c}-6.00 * * \\
(1.24)\end{array}$ & $\begin{array}{l}1.06 * * \\
(0.30)\end{array}$ & $\begin{array}{c}2.65 * * \\
(0.78)\end{array}$ & $\begin{array}{l}1.78^{* * *} \\
(0.49)\end{array}$ \\
\hline Jalna & $\begin{array}{l}0.10^{\mathrm{NS}} \\
(0.84)\end{array}$ & $\begin{array}{c}8.44 * * \\
(2.43)\end{array}$ & $\begin{array}{l}7.22 * \\
(2.18)\end{array}$ & $\begin{array}{l}0.74^{\mathrm{NS}} \\
(0.47)\end{array}$ & $\begin{array}{l}4.23^{*} \\
(1.28)\end{array}$ & $\begin{array}{l}3.67^{*} \\
(1.20)\end{array}$ & $\begin{array}{l}5.77 * * \\
(0.96)\end{array}$ & $\begin{array}{c}-1.42^{\mathrm{NS}} \\
(1.72)\end{array}$ & $\begin{array}{c}-6.45^{* *} \\
(1.19)\end{array}$ & $\begin{array}{c}3.13^{* *} \\
(0.26)\end{array}$ & $\begin{array}{c}4.17 * * \\
(0.47)\end{array}$ & $\begin{array}{c}1.47 * * \\
(0.45)\end{array}$ \\
\hline Beed & $\begin{array}{c}-5.07 * * \\
(0.72)\end{array}$ & $\begin{array}{l}1.28^{\mathrm{NS}} \\
(2.24)\end{array}$ & $\begin{array}{c}6.19 * * \\
(1.76)\end{array}$ & & $\begin{array}{l}4.02^{*} \\
(1.52)\end{array}$ & $\begin{array}{c}-1.03^{\mathrm{NS}} \\
(1.04)\end{array}$ & $\begin{array}{l}3.95^{*} \\
(1.31)\end{array}$ & & & & & \\
\hline Latur & $\begin{array}{l}2.69^{\mathrm{NS}} \\
(1.67)\end{array}$ & $\begin{array}{c}12.79 * * \\
(3.41)\end{array}$ & $\begin{array}{c}10.97 * * \\
(2.32)\end{array}$ & $\begin{array}{c}5.71 * * \\
(1.09)\end{array}$ & $\begin{array}{c}4.57^{N S} \\
(2.44)\end{array}$ & $\begin{array}{c}-0.79^{\mathrm{NS}} \\
(1.64)\end{array}$ & $\begin{array}{c}4.63^{* *} \\
(0.36)\end{array}$ & & $\begin{array}{c}3.65^{\mathrm{NS}} \\
(1.87)\end{array}$ & $\begin{array}{l}5.75^{* * *} \\
(0.24)\end{array}$ & $\begin{array}{c}10.04 * * \\
(0.82)\end{array}$ & $\begin{array}{c}3.55^{* * *} \\
(0.45)\end{array}$ \\
\hline Osmanabad & $\begin{array}{l}0.91^{\mathrm{NS}} \\
(0.97)\end{array}$ & $\begin{array}{l}9.02^{*} \\
(2.84)\end{array}$ & $\begin{array}{l}7.50 * \\
(2.50)\end{array}$ & $\begin{array}{c}4.81 * * \\
(0.63)\end{array}$ & $\begin{array}{c}3.04^{\mathrm{NS}} \\
(2.41)\end{array}$ & $\begin{array}{c}-1.47^{\mathrm{NS}} \\
(2.09)\end{array}$ & $\begin{array}{l}1.42^{\mathrm{NS}} \\
(1.43)\end{array}$ & $\begin{array}{c}-0.52^{\mathrm{NS}} \\
(1.66)\end{array}$ & $\begin{array}{c}-1.56^{\mathrm{NS}} \\
(1.36)\end{array}$ & $\begin{array}{c}3.45^{* * *} \\
(0.26)\end{array}$ & $\begin{array}{l}5.38 * * \\
(0.65)\end{array}$ & $\begin{array}{c}2.24 * * \\
(0.45)\end{array}$ \\
\hline Nanded & $\begin{array}{l}5.23^{*} \\
(1.71)\end{array}$ & $\begin{array}{c}13.48^{* *} \\
(1.99)\end{array}$ & $\begin{array}{c}7.46^{* *} \\
(1.15)\end{array}$ & $\begin{array}{c}5.89 * * \\
(1.30)\end{array}$ & $\begin{array}{c}11.53 * * \\
(1.52)\end{array}$ & $\begin{array}{l}5.22 * * \\
(1.21)\end{array}$ & $\begin{array}{l}1.03^{\mathrm{NS}} \\
(0.95)\end{array}$ & $\begin{array}{l}3.44^{\mathrm{NS}} \\
(2.13)\end{array}$ & $\begin{array}{l}1.76^{\mathrm{NS}} \\
(1.98)\end{array}$ & $\begin{array}{c}5.30 * * \\
(0.32)\end{array}$ & $\begin{array}{l}8.34 * * \\
(0.74)\end{array}$ & \\
\hline Parbhani & $\begin{array}{l}0.56^{\mathrm{NS}} \\
(0.77)\end{array}$ & $\begin{array}{l}7.71 * \\
(1.83)\end{array}$ & $\begin{array}{c}6.92 * * \\
(1.22)\end{array}$ & $\begin{array}{c}-1.27^{\mathrm{NS}} \\
(1.35) \\
\end{array}$ & $\begin{array}{c}-1.76^{\mathrm{NS}} \\
(1.36)\end{array}$ & $\begin{array}{c}-0.66^{\mathrm{NS}} \\
(1.20)\end{array}$ & $\begin{array}{c}-0.21^{\mathrm{NS}} \\
(0.89)\end{array}$ & $\begin{array}{c}-1.92^{\mathrm{NS}} \\
(1.27)\end{array}$ & $\begin{array}{c}-1.89 * \\
(0.65)\end{array}$ & $\begin{array}{l}1.53 * * \\
(0.22)\end{array}$ & $\begin{array}{l}3.10 * * \\
(0.36)\end{array}$ & $\begin{array}{l}1.60 * * \\
(0.28)\end{array}$ \\
\hline Hingoli & NA & NA & NA & $\begin{array}{c}7.79 * * \\
(1.33)\end{array}$ & $\begin{array}{c}12.04 * * \\
(1.40)\end{array}$ & $\begin{array}{l}4.02 * \\
(1.17)\end{array}$ & $\begin{array}{c}13.30^{* * *} \\
(2.44)\end{array}$ & $\begin{array}{c}32.28^{* *} \\
(7.18)\end{array}$ & $\begin{array}{c}9.77 * * \\
(2.90)\end{array}$ & $\begin{array}{c}10.61 * * \\
(1.16)\end{array}$ & $\begin{array}{c}28.95^{* *} \\
(4.51)\end{array}$ & $\begin{array}{l}7.49^{* *} \\
(1.22)\end{array}$ \\
\hline Marathwada & $\begin{array}{l}1.38^{\mathrm{NS}} \\
(0.89)\end{array}$ & $\begin{array}{c}8.48^{* *} \\
(2.43)\end{array}$ & $\begin{array}{c}7.31 * * \\
(1.80)\end{array}$ & $\begin{array}{c}3.98 * * \\
(0.44)\end{array}$ & $\begin{array}{c}4.38 * * \\
(1.08)\end{array}$ & $\begin{array}{l}0.28^{\mathrm{NS}} \\
(1.00)\end{array}$ & $\begin{array}{l}2.79 * * \\
(0.54)\end{array}$ & $\begin{array}{c}3.90 * * \\
(0.71)\end{array}$ & $\begin{array}{c}0.77^{\mathrm{NS}} \\
(0.59)\end{array}$ & $\begin{array}{c}4.19 * * \\
(0.16)\end{array}$ & $\begin{array}{l}7.15^{* *} \\
(0.39)\end{array}$ & $\begin{array}{c}2.70 * * \\
(0.29)\end{array}$ \\
\hline Maharashtra & $\begin{array}{l}3.04^{*} \\
(0.94)\end{array}$ & $\begin{array}{c}9.39 * * \\
(2.09)\end{array}$ & $\begin{array}{l}6.71 * * \\
(1.37)\end{array}$ & $\begin{array}{l}1.59^{\mathrm{NS}} \\
(0.72)\end{array}$ & $\begin{array}{l}1.15^{\mathrm{NS}} \\
(1.06)\end{array}$ & $\begin{array}{c}-0.59^{\mathrm{NS}} \\
(0.70)\end{array}$ & $\begin{array}{l}0.63^{\mathrm{NS}} \\
(0.600\end{array}$ & $\begin{array}{l}1.60^{* * *} \\
(0.46)\end{array}$ & $\begin{array}{c}0.94^{\mathrm{NS}} \\
(0.63)\end{array}$ & $\begin{array}{c}3.79 * * \\
(0.22)\end{array}$ & $\begin{array}{l}6.16^{* *} \\
(0.43)\end{array}$ & $\begin{array}{c}2.35^{* *} \\
(0.24)\end{array}$ \\
\hline
\end{tabular}

Note: Figures in brackets indicate standard error, ${ }^{*}$ Significant at 5 per cent level, ${ }^{* *}$ Significant at 1 per cent level, NS- Non significant 
Annexure.1 Area dynamics in pulse crops of Marathwada region of Maharashtra State

\begin{tabular}{|c|c|c|c|c|c|}
\hline Districts & 1986-95 & 1996-05 & $2006-15$ & $\begin{array}{c}\% \text { change in 1996-05 } \\
\text { over base }\end{array}$ & $\begin{array}{c}\% \text { change in } 2006-15 \\
\text { over base }\end{array}$ \\
\hline Aurangabad & 440 & 414 & 397 & 94.09 & 90.22 \\
\hline Jalna & 391 & 462 & 477 & 118.15 & 121.99 \\
\hline Beed & 492 & 480 & 560 & 97.56 & 113.82 \\
\hline Latur & 632 & 715 & 690 & 113.13 & 109.17 \\
\hline Osmanabad & 717 & 778 & 875 & 108.50 & 122.03 \\
\hline Nanded & 448 & 521 & 654 & 116.29 & 145.98 \\
\hline Parbhani & 694 & 611 & 646 & 88.04 & 93.08 \\
\hline Hingoli & NA & 252 & 339 & 252 & 339 \\
\hline Marathwada & 3815 & 4148 & 4908 & 108.72 & 128.65 \\
\hline Maharashtra & 9516 & 10502 & 11720 & 110.36 & 123.16 \\
\hline
\end{tabular}

Note: Base year was 1986-95 
During second sub period, many of the districts shown negative trends in area of pigeon pea crop. Only few districts viz; Jalna, Osmanabad, Nanded districts have shown positive trend in area of pigeon pea crop. During third sub period, many of the districts shown positive trends in area of pigeon pea crop except Aurangabad and Jalna districts. In overall period, except Aurangabad and Parbhani districts all districts have shown positive trend. It was statistically significant at 1 per cent level of significance. Magnitude of CGR in regards to production during the first sub period, the highest CGR of pigeon pea production was showed in Aurangabad i.e. 3.82 per cent per annum. During overall period, production of pigeon pea crop was seen high in Latur district (6.62 per cent per annum). In case of productivity, growth rate was found to be highest during overall period in Latur district it was 4.30 per cent per annum.

\section{Chickpea}

The annual compound growth rate (CGR) in area of chickpea crop was 1.38, 3.98, 2.79 and 4.19 per cent per annum during three sub periods and overall period at Marathwada region of Maharashtra State level, respectively (Table 2). It was statistically significant at 1 per cent level of significance (except first sub period). This shows that, maximum growth in area of chickpea crop has been occurred during second sub period (1996-97 to 2005-06). Yield of chickpea has shown mixed trend in all the three sub period of study. Maximum growth in yield of chickpea was observed during first sub period. Similar results were reported by Shimar (2014). During overall period productivity under chickpea crop was 2.70 per cent per annum. It was statistically significant at 1 per cent level of significance. Positive and significant trend in production of chickpea crop was also seen in overall period. During this first sub period CGR of production was highest i.e. 8.48 per cent per annum. The CGR of production during three sub periods were $8.48,4.38$ and 3.90 per cent, respectively.

The district level results shows that, in first sub period of study (1986-87 to 1995-96), all the districts has shown positive trend in area of chickpea crop (except Beed district) but high CGR i.e. more than 3 per cent was recorded in Aurangabad and Nanded districts. During second sub period, many of the districts have shown positive trends in area of chickpea crop (except Aurangabad and Parbhani district). During third sub period, many of the districts have shown positive trends in area of chickpea crop (except Aurangabad and Parbhani districts). In overall period, all districts have shown positive trend. It was statistically significant at 1 per cent level of significance. The highest CGR of chickpea production was showed in Nanded district i.e. 13.48 per cent per annum. During overall period, production of chickpea was seen high in Hingoli district (28.95 per cent per annum). In case of productivity, growth rate was found to be highest in during overall period in Hingoli district i.e. 7.49 per cent per annum Chaudhari and Pawar(2010).

In conclusion, the results indicated that, average area, production and productivity of pigeon pea and chickpea in the district, region and state level was continuously increased during study period except in Aurangabad district. In Aurangabad districts, the average area of pigeon pea was reduced. The growth in area, production and productivity of pigeon pea and chickpea was positive and significant during overall study period except in Aurangabad and Parbhani district. Consistent improvement in the yield of pulses was a notable feature which shows that improved technology and implementation of government programme has payoff in the state. 


\section{References}

Asthurkar, B.W. and Kohal, A.N. (1993). Performance of tur in Maharashtra. 1960-61 to 1990-91, Indian Journal Agriculture Economics, 45(3): 434-436.

Brahmprakash, J.V. and Sushila, Shrivastava. (1998). Regional variation in performance of pulses, Indian Journal Agriculture Economics, 52(3): 424-425.

Chaudhari, D.J. and Pawar, N.D. (2010). Growth, instability and price analysis of pigeon pea in Marathwada region, Agriculture Update, 5(1\&2): 158-162.

Chavan, R., Patil, S.S., Jainuddin, S.M. and Yasmeen. (2017). Growth, Demand and Supply of Quality Seeds of Pulses in India, Indian Journal of Economics and Development, 13(2a): 676-679.

Devaraj, Jha, G.K. and Khare, A.P. (2006).Analysis of growth and instability of chickpea (gram) production in Madhya Pradesh, Agriculture Situation in India, 63 (4): 251-259.

More, S.S., Narendra Singh and Bhatt, B.K. (2018). Performance of pulses in Gujarat: A district level Assessment,
Current Agriculture Research Journal, 6(1): 45-53.

More, S.S., Singh, N. and Kuthe, S.B. (2015). Performance of Pulses Crops in Gujarat State: A Decomposition Analysis, International Journal of Agriculture Sciences, 7(5): 510-515.

Narayan, P. and Kumar, S. (2015). Constraints of growth in area production and productivity of pulses in India: An analytical approach to major pulses, Indian Journal Agriculture Research., 49(2): 114-124.

Reddy, A.A. (2005). Growth and instability in chickpea production in India: A state level analysis, Agriculture Situation in India, 62: 621-629

Shimar, R. (2014). Growth and Instability in Agricultural Production in Haryana: A District level Analysis, International journal of Scientific and Research Publications, 4(7): 1-12.

Shingne, S.P., Shende, N.V., Panajwar, A.V., Rathod, S.A. and Raut, N.V. (2017). Performance of Gram in Marathwada Region, International journal of Information research and Review, 6(2): 24-27.

\section{How to cite this article:}

Dhokar, N.R., S.S. More and Shelke, R.D. 2018. Growth Analysis of Pigeon Pea and Chickpea in Marathwada Region of Maharashtra State, India. Int.J.Curr.Microbiol.App.Sci. 7(08): 20142021. doi: https://doi.org/10.20546/ijcmas.2018.708.232 